\title{
Differential expression of filamin A and its clinical significance in breast cancer
}

\author{
HUI-MIN TIAN ${ }^{1}$, XIU-HUA LIU $^{1}$, WEI HAN ${ }^{2}$, LING-LING ZHAO $^{1}$, BO YUAN $^{3}$ and CHANG-JI YUAN ${ }^{1}$ \\ ${ }^{1}$ Cancer Center; ${ }^{2}$ Department of Anesthesia, The First Hospital of Jilin University; \\ ${ }^{3}$ Norman Bethune College of Medicine, Jilin University, Changchun, Jilin 130021, P.R. China
}

Received April 1, 2012; Accepted May 1, 2013

DOI: $10.3892 / \mathrm{ol} .2013 .1454$

\begin{abstract}
Changes in filamin A (FLNa) expression contribute to the development and progression of numerous malignancies. However, in vitro studies of breast cancer have shown conflicting results. Thus, the present study aimed to detect the expression of FLNa in breast cancer tissue samples and the association with clinicopathological data, in order to provide insightful ex vivo data. A total of 96 breast cancer and distant normal breast tissues and 20 benign tumor tissue specimens were subjected to immunohistochemistry or reverse transcription polymerase chain reaction (RT-PCR) analysis of FLNa expression. Clinicopathological data were collected to analyze the association with FLNa expression. The FLNa protein was overexpressed in breast cancer tissues compared with distant normal mammary gland and benign breast tissues. The FLNa protein was expressed in $63.5 \%$ of breast cancer, with positive rates of $36,66.7$ and $84.6 \%$, respectively, in stage I, II and III breast cancer patients $(\mathrm{P}<0.05)$. Overexpression of the FLNa protein was associated with advanced stage, lymph node metastasis, vascular or neural invasion, menstruation state and other risk stratifications for breast cancer. The overexpression of FLNa in breast cancer was validated by RT-PCR, indicating transcriptional regulation of FLNa overexpression in breast cancer. FLNa mRNA and protein were overexpressed in breast cancer tissues, which was associated with advanced stage, lymph node metastasis and vascular or neural invasion of breast cancer, suggesting that FLNa contributes to breast cancer development and progression.
\end{abstract}

\section{Introduction}

Breast cancer is the most significant worldwide health problem in women $>35-40$ years of age. Additionally, breast

Correspondence to: Professor Chang-Ji Yuan, Cancer Center, The First Hospital, Jilin University, No. 71 Xinmin Street, Changchun, Jilin 130021, P.R. China

E-mail: yuanchangji@163.com

Key words: breast cancer, filamin A, immunohistochemistry, reverse transcription-polymerase chain reaction cancer accounts for $\sim 1.35$ million new cases and $>450,000$ cancer-related mortalities annually worldwide (1). Despite improved early detection, treatment options and survival, the morbidity and mortality continue to increase and numerous patients with invasive breast cancer develop metastatic diseases that eventually lead to the patient succumbing to their condition. Thus, there is an urgent requirement to search for and identify novel biomarkers to predict tumor recurrence and metastasis and to develop more novel treatment strategies to effectively control aggressive breast cancers.

To this end, the present study focused on the identification of biomarkers for breast cancer. One possible biomarker of breast cancer is filamin A (FLNa), which is a cytoskeletal protein with a molecular weight of $280 \mathrm{kDa}(2,3)$ that crosslinks actin filaments into orthogonal networks. FLNa also interacts with $>45$ proteins and serves as the scaffold in various signaling networks $(4,5)$. The FLNa protein, containing an integrin- $\beta$ binding domain and an RAC1 binding domain, is localized in the edge of the cytoplasm, is able to cross the membrane and may even appear in the nuclei $(6,7)$. The actin cytoskeleton is central to numerous cell functions, including the maintenance of cell shape, cell division, adhesion, motility, signal transduction and protein sorting. The alteration of FLNa expression may contribute to cancer development and progression $(4,5,8,9)$, and previous studies have identified FLNa overexpression in a number of malignancies (10-12). For example, in lung cancer, FLNa overexpression was shown to be associated with tumor metastasis (11), and in melanoma, FLNa-positive cells had higher migration and invasion abilities compared with FLNa-negative tumor cells (12). In breast cancer, it was reported that cyclin D1 interacted with the FLNa protein to affect the migration and invasion potential of breast cancer cells (13). However, another study showed that FLNa was able to regulate focal adhesion disassembly and suppress breast cancer cell migration and invasion (14). The reason for this discrepancy or conflict in the data is unclear. To date, there have been no studies reporting FLNa expression in ex vivo breast cancer tissue specimens; thus, the present study was proposed in order to detect FLNa expression in breast cancer tissue samples and to identify any associations between FLNa expression and clinicopathological data. The present results may provide useful information with regard to the function of FLNa in breast cancer and the potential of FLNa as a biomarker to predict breast cancer progression. 


\section{Materials and methods}

Tumor tissue samples. Tissue samples were recruited from 96 consecutive primary breast cancer patients who underwent surgical resection between January 2008 and January 2010 at the Department of Breast Surgery, First Hospital of Jilin University (Changchun, Jilin, China). None of these patients received chemotherapy, radiotherapy or immunotherapy prior to surgery. All the hematoxylin and eosin-stained tissue sections were re-evaluated and confirmed by two pathologists according to the World Health Organization classifications (NCCN Breast Cancer Guidelines, version 1, 2013). Of the 96 samples, 82 cases were classified as invasive breast cancer and 14 as non-invasive cancer. Paraffinized and snap-frozen tissues of distant normal breast tissue and 20 benign tumors were also included in the study as controls. Normal skin tissues were obtained from the healthy skin of the chest area of female patients who underwent benign tumor resections and were used as a positive control for FLNa expression. In addition, 30 cases of breast cancer and distant non-tumor specimens were snap-frozen in liquid nitrogen and stored at $-80^{\circ} \mathrm{C}$ after resection for reverse transcription PCR (RT-PCR) analysis. The institutional review board of Jilin University approved the study and each patient signed a consent form agreeing to their participation in the study.

Immunohistochemical staining. The formalin-fixed and paraffin-embedded tissue sections were prepared for immunohistochemical analysis of FLNa protein expression. Briefly, archival paraffin blocks were retrieved and $3-\mu \mathrm{m}$ thick tissue sections were prepared. For immunohistochemistry, the sections were deparaffinized in xylene and rehydrated in a series of graded ethanol solutions. The sections were immersed in citrate buffer $(0.01 \mathrm{~mol} / \mathrm{l}$ citric acid, $\mathrm{pH} 6.0)$ and heated for two $5 \mathrm{~min}$ intervals in a microwave oven for antigen retrieval. Next, the sections were incubated with $0.3 \% \mathrm{H}_{2} \mathrm{O}_{2}$ for $15 \mathrm{~min}$ to block potential endogenous peroxidase activity. Subsequent to the sections being rinsed in tap water and washed with phosphate-buffered saline (PBS), the sections were incubated with $20 \%$ normal goat serum for $30 \mathrm{~min}$ at room temperature, then incubated with a monoclonal mouse anti-FLNa antibody (Millipore, Bedford, MA, USA; 1:100) in PBS for $18 \mathrm{~h}$ at $4^{\circ} \mathrm{C}$. The next day, the sections were washed with PBS three times, then incubated with a goat anti-mouse polymer secondary antibody (Zhongshan Goldenbridge Biotechnology Co., Ltd., Beijing, China) for $30 \mathrm{~min}$ at room temperature. Next, DAB plus (Zhongshan Goldenbridge Biotechnology Co.) was added to the sections once they had briefly been washed with PBS and incubated for 5 min to visualize the positive signal. Finally, the sections were counterstained with hematoxylin. Normal skin tissue sections served as the positive control, while normal mammary gland and breast benign tumor tissues served as the negative controls. PBS was used as a blank control instead of the first antibody. The stained tissue sections were reviewed and scored under a light microscope: the intensity of staining was scored as zero (no staining); $1+$ (weak cytoplasmic staining in $<10 \%$ of cells); $2+$ (moderate cytoplasmic staining in $>10 \%$ of cells); and $3+$ (marked cytoplasmic staining in $>10 \%$ of the cells). A score of 0 or 1 was considered to indicate a negative result for FLNa expression (low), whereas scores of $2+$ or $3+$ were considered to show positive (high) FLNa expression.

$R T-P C R$. An RT-PCR analysis was performed to detect FLNa mRNA expression in the snap-frozen breast cancer and distant normal breast tissues. Total RNA from these tissues was isolated using TRIzol reagent (Invitrogen, Carlsbad, CA, USA) according to the manufacturer's instructions. After measuring the quantity and quality using a spectrophotometer, the total RNA was subjected to reverse transcription into cDNA with $1 \mu \mathrm{g}$ RNA as a template, using an RT kit (Fermentas, Glen Burnie, MD, USA) in a total volume of $20 \mu \mathrm{l}$. PCR amplification was then performed using primers derived from the human FLNa sequence (5'-AGCCTCCACGAGACATCATC-3' and 5'-CCAGTGTGT ACTCCCCCTTG-3'). The results were normalized to GAPDH, which was used as an internal control. The primer sequences of GAPDH were 5'-GGGTGATGCTGGTGCTGA GTATGT-3' and 5'-AAGAATGGGAGTTGCTGTTGA AGTC-3'. A semi-quantitative determination of the FLNa mRNA levels was achieved following 30 cycles of PCR amplification. The PCR products were then analyzed using $1 \%$ ethidium bromide agarose gel electrophoresis in comparison with the DNA molecular weight marker (Takara, Dalian, China). Finally, a quantitative analysis of the PCR target bands was performed using Image J software (NIH, Bethesda, MD, USA).

Statistical analysis. SPSS software version 13.0 for Windows (SPSS Inc., Chicago, IL, USA) was used for the statistical analyses. Two-sample t-tests or Mann-Whitney U tests, with respect to data distribution in the measured data, were performed for comparisons between two groups. $\chi^{2}$ tests were used for enumeration data. $\mathrm{P}<0.05$ was considered to indicate a statistically significant difference.

\section{Results}

Overexpression of FLNa protein in breast cancer tissues. First, FLNa protein expression was assessed in the breast cancer and distant normal and benign breast tumor tissue specimens (Fig. 1). The data showed that the FLNa protein was mainly detected in the cytoplasm of the breast cancer cells, mostly at the edge of the cells and in the basal cells or intercellular substance (Fig. 1 and 2).

The expression of the FLNa protein was detected in $63.54 \%$ of the breast cancer tissues, whereas the immunoreactivity of the FLNa antibody was extremely low in certain distant normal tissues. FLNa was undetectable in the most distant normal breast tissues and benign tumor sections (Fig. 2). Furthermore, in the breast cancer samples, the positive rate of FLNa staining was increased according to the tumor stage, with 36, 66.7 and $84.6 \%$ in stage I, II and III breast cancer tissues, respectively $(\mathrm{P}<0.05)$.

Association of FLNa protein expression with clinicopathological features of patients with breast cancer. Next, the associations between FLNa protein expression and the clinicopathological features of the patients with breast cancer were investigated. It was observed that the expression of the FLNa 

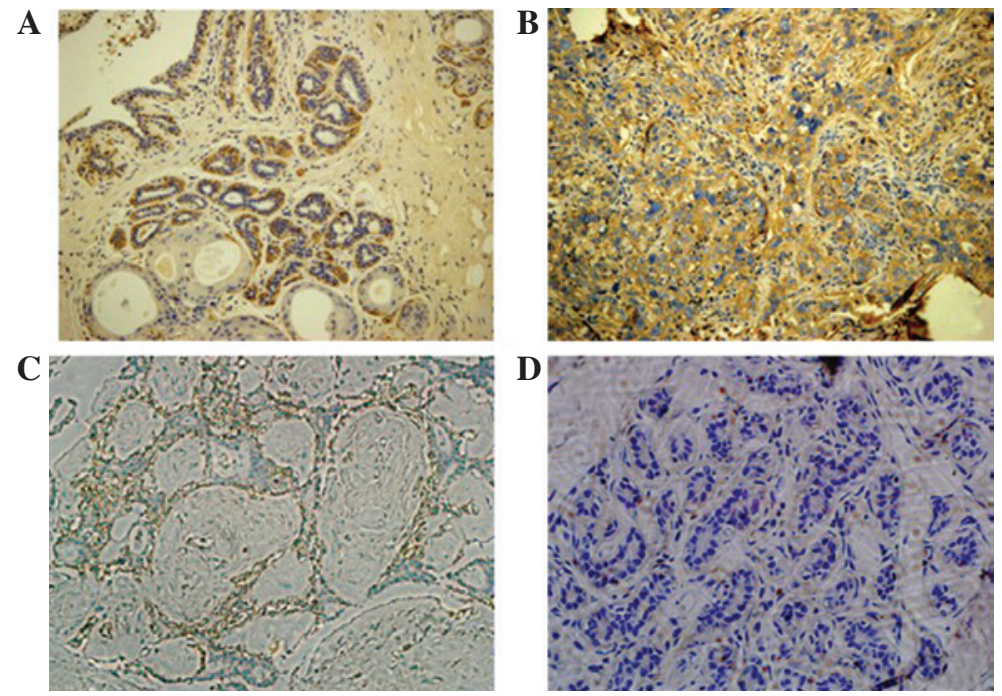

Figure 1. Differential expression of FLNa protein in breast cancer tissue specimens. Immunohistochemical analysis of FLNa protein expression in (A) non-invasive and (B) invasive breast cancer tissues and (C) benign breast tumor and (D) distant normal mammary gland tissues. All images are at x200 magnification. FLNa, filamin A.
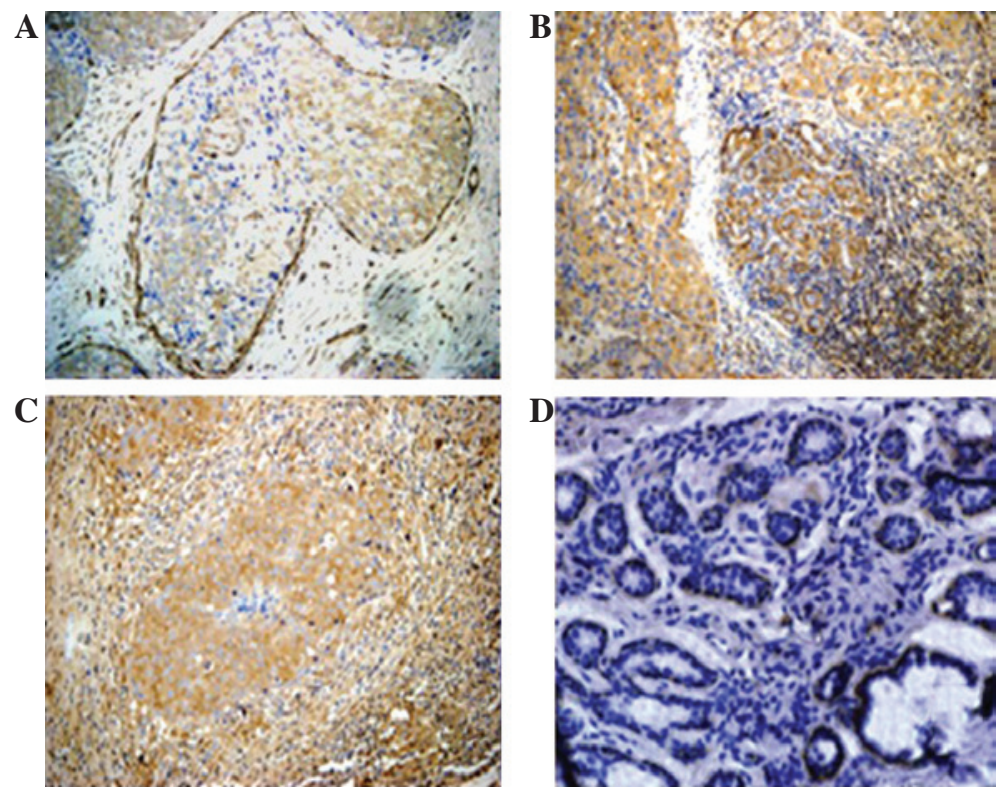

Figure 2. Expression of FLNa protein in breast cancer tissues. Immunohistochemical staining of FLNa protein in (A) Stage I, (B) II and (C) III breast cancer tissues compared with (D) normal breast tissue. All images are at x200 magnification. FLNa, filamin A.

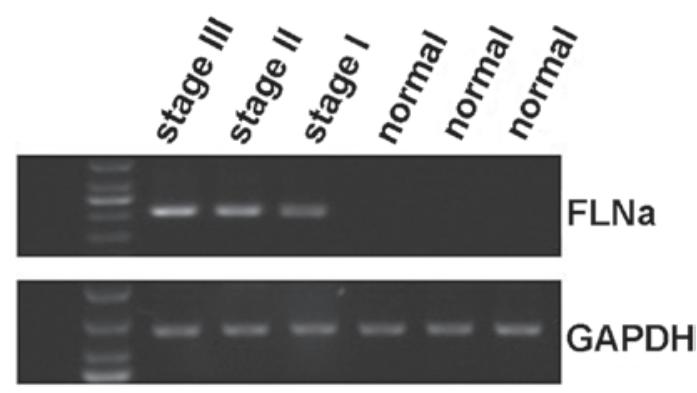

Figure 3. Representative semi-quantitative reverse transcription polymerase chain reaction (RT-PCR) data on FLNa mRNA levels in breast cancer (stages I, II and III) vs. the distant non-tumor tissues. Detection of GAPDH mRNA served as an internal control. FLNa, filamin A. protein was associated with TNM stage, lymph node metastasis, vascular or neural invasion of the tumors, menstruation state and other risk stratifications $(\mathrm{P}<0.05)$. However, the expression of the FLNa protein was not associated with any other clinicopathological features, including age, tumor size, localization, histological type and the status of the estrogen and progesterone receptors or the Her $2 /$ neu protein $(\mathrm{P}>0.05$; Table I).

Differential expression of FLNa mRNA in breast cancer and distant non-tumor breast tissues. To investigate whether FLNa expression was regulated at the transcriptional level, a semi-quantitative RT-PCR analysis of FLNa mRNA expression was performed on 30 cases of breast cancer and distant normal tissues. After normalizing to the GAPDH mRNA levels, the 
Table I. Association of FLNa protein expression with the clinicopathological characteristics of patients with breast cancer.

\begin{tabular}{|c|c|c|c|c|c|}
\hline Characteristic & $\mathrm{n}$ &,$+ \mathrm{n}$ &,$- \mathrm{n}$ & $\chi^{2}$ & P-value \\
\hline \multicolumn{6}{|l|}{ Age (years) } \\
\hline$\leq 35$ & 5 & 4 & 1 & \multirow[t]{3}{*}{3.154} & \multirow[t]{3}{*}{0.207} \\
\hline $35-55$ & 68 & 45 & 23 & & \\
\hline$\geq 55$ & 23 & 11 & 22 & & \\
\hline \multicolumn{6}{|l|}{ Tumor size $(\mathrm{cm})$} \\
\hline$\leq 2$ & 44 & 23 & 21 & \multirow[t]{3}{*}{4.76} & \multirow[t]{3}{*}{0.120} \\
\hline $2-5$ & 49 & 34 & 15 & & \\
\hline$\geq 5$ & 3 & 3 & 0 & & \\
\hline \multicolumn{6}{|l|}{ Tumor location } \\
\hline Left & 46 & 33 & 13 & \multirow[t]{2}{*}{3.217} & \multirow[t]{2}{*}{0.073} \\
\hline Right & 50 & 27 & 23 & & \\
\hline \multicolumn{6}{|l|}{ TNM stage } \\
\hline I & 25 & 9 & 16 & \multirow[t]{3}{*}{13.360} & \multirow[t]{3}{*}{0.010} \\
\hline II & 45 & 30 & 15 & & \\
\hline III & 26 & 22 & 4 & & \\
\hline \multicolumn{6}{|c|}{ Lymph-node-metastasis } \\
\hline Yes & 53 & 40 & 13 & \multirow[t]{2}{*}{8.495} & \multirow[t]{2}{*}{0.040} \\
\hline No & 43 & 20 & 23 & & \\
\hline \multicolumn{6}{|c|}{ Vascular/nerve infiltration } \\
\hline Yes & 42 & 31 & 11 & \multirow[t]{2}{*}{4.710} & \multirow[t]{2}{*}{0.030} \\
\hline No & 52 & 27 & 25 & & \\
\hline \multicolumn{6}{|l|}{ Pathological type } \\
\hline Invasive & 82 & 52 & 30 & \multirow[t]{2}{*}{0.201} & 0.645 \\
\hline Non-invasive & 14 & 8 & 6 & & \\
\hline Histological type & & & & & \\
\hline I & 3 & 2 & 1 & 0.998 & 1.000 \\
\hline II & 47 & 29 & 18 & & \\
\hline III & 19 & 12 & 7 & & \\
\hline Others & 27 & 17 & 10 & & \\
\hline Menstrual state & & & & & \\
\hline Non-pausimenia & 60 & 42 & 18 & 4.313 & 0.038 \\
\hline Pausimenia & 35 & 17 & 18 & & \\
\hline ER & & & & & \\
\hline- & 32 & 21 & 11 & 0.316 & 0.574 \\
\hline+ & 62 & 37 & 25 & & \\
\hline PR & & & & & \\
\hline- & 43 & 29 & 14 & 1.105 & 0.293 \\
\hline+ & 51 & 29 & 22 & & \\
\hline CerB-b2 & & & & & \\
\hline 0 & 60 & 36 & 24 & 0.216 & 0.975 \\
\hline $1+$ & 3 & 2 & 1 & & \\
\hline $2+$ & 11 & 7 & 4 & & \\
\hline $3+$ & 20 & 13 & 7 & & \\
\hline Risk & & & & & \\
\hline Low & 6 & 2 & 4 & 13.426 & 0.010 \\
\hline Middle & 56 & 29 & 27 & & \\
\hline High & 34 & 29 & 5 & & \\
\hline MBNG & & & & & \\
\hline I & 10 & 6 & 4 & 0.725 & 0.867 \\
\hline II & 49 & 32 & 17 & & \\
\hline III & 25 & 14 & 11 & & \\
\hline Others & 12 & 8 & 4 & & \\
\hline
\end{tabular}

Modified Black's nuclear grade (MBNG) is a nuclear classification of breast cancer cells. ER, estrogen receptor; PR, progesterone receptor; FLNa, filamin A. 
expression level of the FLNa mRNA was $0.634 \pm 0.53$ in the breast cancer tissues and $0.06 \pm 0.01$ in the distant non-tumor breast tissues $(\mathrm{P}<0.05$; Fig. 3).

\section{Discussion}

In the present study, the differential expression of FLNa mRNA and protein was analyzed in breast cancer tissue specimens in order to provide ex vivo data on the potential role of the FLNa protein in breast cancer. It was observed that the FLNa protein was overexpressed in the breast cancer tissues compared with the distant normal mammary gland and benign breast tissues, and that this overexpression was associated with advanced stages, lymph node metastasis and vascular or neural invasion of breast cancer. Semi-quantitative RT-PCR data showed the transcriptional regulation of FLNa overexpression in breast cancer. The present data indicate that FLNa contributes to breast cancer development and progression.

In the present study, FLNa expression was demonstrated in the cytoplasm of the breast cancer cells, mainly at the edge of the cells and in the basal cells or intercellular substance. Until now, it was difficult to determine the association between the function and the localization of FLNa. However, we provide clinical evidence demonstrating that FLNa is frequently overexpressed in breast cancer specimens.

Various other studies have demonstrated an association between FLNa overexpression and tumor metastasis in several types of cancer. For example, the expression of FLNa protein was higher in hepatocellular carcinoma HCCLM9 cells with high metastatic potential compared with hepatocellular carcinoma MHCC97L cells with low metastatic potential (10). The overexpression of FLNa has been associated with the invasion and metastasis of breast cancer (15). Furthermore, FLNa has been shown to affect the invasion and metastatic capacity of lung tumor cells (11). FLNa was reported to be essential for the locomotion of human melanoma cells, and the suppression of FLNa expression inhibited melanoma cell migration and induced apoptosis $(12,16)$. The present study supported these published data. However, two further studies showed contradicting data. The first study by Zhong et al reported that the knockdown of cyclinD1 expression suppressed breast cancer cell invasion, which was associated with the downregulation of FLNa protein phosphorylation (13). The second study by Xu et al showed that FLNa suppressed the migration and invasion capacity of breast cancer (14). The present ex vivo data supported the hypothesis that FLNa expression is associated with breast cancer development and progression. Structurally and molecularly, the FLNa protein functions to crosslink actin filaments into orthogonal networks and serves as the scaffold in various signaling networks $(4,5)$, which in turn has a role in regulating cell shape, adhesion and motility. During tumorigenesis, FLNa may regulate tumor cell invasion and metastasis. Cancer metastasis is, however, a major obstacle for cancer therapy and targeting it may effectively control advanced or aggressive tumors. In addition, the development of novel biomarkers to predict tumor progression, such as metastasis, may also improve cancer survival and reduce fatalities. These biomarkers should distinguish cancers with high metastatic potential from cancers with less metastatic potential, thus optimizing individualized therapeutic planning. However, whether the detection of FLNa protein expression is likely to service as such a biomarker requires further study and verification. Indeed, an additional cohort of tissue samples is likely to aid in the confirmation of the current data.

Previous studies have shown that FLNa expression is positively associated with VEGF, an angiogenesis regulator, in lung cancer (11). FLNa is an important regulatory molecule in the TGF- $\beta$ signal transduction pathways (17) and is able to bind to SMAD2 to regulate actin polymerization reconciliation and interaction with myosin via $\beta_{1}$-integrin and RhoA GTPase. Kim et al (18) reported that FLNa and $\beta_{1}$-integrin interacted together to mediate lung cancer (A549) cell proliferation and prevent apoptosis. In addition, FLNa was reported to interact with CEACAM1 (19), P311 (20) and FilGAP (21) to promote tumor cell migration. Ravid et al (22) showed that caveolin-1 expression in breast cancer MCF-7 cells upregulated FLNa phosphorylation to induce MCF-7 cell migration through the PI3/AKT pathway. The FLNa protein also interacted with the GTP-binding protein R-Ras to promote metastasis of melanoma cells (23). Together, these findings and the results of the present study clearly support the hypothesis that FLNa is involved in tumor invasion and metastasis. However, it has also been reported that FLNa is able to inhibit MMP-9 expression through Ras/MAPK/ERK signaling to affect tumor cell invasion (24). Thus, future studies should further investigate the role of FLNa in human carcinogenesis and cancer progression.

\section{Acknowledgements}

The authors would like to thank Medjaden Bioscience Limited (Hong Kong, SAR, China) for assisting in the preparation of the original manuscript.

\section{References}

1. Qiang S: The early diagnosis of breast cancer. J Practical Med 23: 165-168, 2007 (In Chinese)

2. Wang K, Ash JF and Singer SJ: Filamin, a new high-molecular-weight protein found in smooth muscle and non-muscle cells. Proc Natl Acad Sci USA 72: 4483-4486, 1975.

3. Wang K: Filamin, a new high-molecular-weight protein found in smooth muscle and nonmuscle cells. Purification and properties of chicken gizzard filamin. Biochemistry 16: 1857-1865, 1977.

4. Feng Y and Walsh CA: The many faces of filamin: a versatile molecular scaffold for cell motility and signalling. Nat Cell Biol 6: 1034-1038, 2004.

5. Popowicz GM, Schleicher M, Noegel AA and Holak TA: Filamins: promiscuous organizers of the cytoskeleton. Trends Biochem Sci 31: 411-419, 2006.

6. Bachmann AS, Howard JP and Vogel CW: Actin-binding protein filamin A is displayed on the surface of human neuroblastoma cells. Cancer Sci 97: 1359-1365, 2006.

7. Zhou X, Borén J and Akyürek LM: Filamins in cardiovascular development. Trends Cardiovasc Med 17: 222-229, 2007.

8. Robertson SP, Twigg SR, Sutherland-Smith AJ, et al: Localized mutations in the gene encoding the cytoskeletal protein filamin A cause diverse malformations in humans. Nat Genet 33: 487-491, 2003.

9. Keshamouni VG, Michailidis G, Grasso CS, et al: Differential protein expression profiling by iTRAQ-2DLC-MS/MS of lung cancer cells undergoing epithelial-mesenchymal transition reveals a migratory/invasive phenotype. J Proteome Res 5: 1143-1154, 2006. 
10. Ai J, Huang H, Lv X, et al: FLNA and PGK1 are two potential markers for progression in hepatocellular carcinoma. Cell Physiol Biochem 27: 207-216, 2011.

11. Uramoto H, Akyurek LM and Hanagiri T: A positive relationship between filamin and VEGF in patients with lung cancer. Anticancer Res 30: 3939-3944, 2010.

12. Flanagan LA, Chou J, Falet H, Neujahr R, Hartwig JH and Stossel TP: Filamin A, the Arp2/3 complex, and the morphology and function of cortical actin filaments in human melanoma cells. J Cell Biol 155: 511-517, 2001.

13. Zhong Z, Yeow WS, Zou C, et al: Cyclin D1/cyclin-dependent kinase 4 interacts with filamin $\mathrm{A}$ and affects the migration and invasion potential of breast cancer cells. Cancer Res 70 : 2105-2114, 2010

14. Xu Y, Bismar TA, Su J, et al: Filamin A regulates focal adhesion disassembly and suppresses breast cancer cell migration and invasion. J Exp Med 207: 2421-2437, 2010.

15. Wu Y, Li J, Zhao R, Wang X, Shan B and Zhu T: Expression of filamin A in invasive breast carcinoma and its significance. Tumor 29: 659-662, 2009 (In Chinese).

16. Cunningham CC, Gorlin JB, Kwiatkowski DJ, et al: Actin-binding protein requirement for cortical stability and efficient locomotion. Science 255: 325-327, 1992.

17. Wang J, Fan Y, Guo L and Lu S: Search for interacting proteins of esophageal cancer related gene-1 encoded protein through the yeast two-hybrid system. Zhonghua Zhong Liu Za Zhi 24: 219-221, 2002 (In Chinese).
18. Kim H, Sengupta A, Glogauer M and McCulloch CA: Filamin A regulates cell spreading and survival via betal integrins. Exp Cell Res 314: 834-846, 2008

19. Klaile E, Muller MM, Kannicht C, Singer BB and Lucka L: CEACAM1 functionally interacts with filamin A and exerts a dual role in the regulation of cell migration. J Cell Sci 118: 5513-5524, 2005.

20. McDonough WS, Tran NL and Berens ME: Regulation of glioma cell migration by serine-phosphorylated P311. Neoplasia 7: 862-872, 2005

21. Ohta Y, Hartwig JH and Stossel TP: FilGAP, a Rho- and ROCK-regulated GAP for Rac binds filamin A to control actin remodelling. Nat Cell Biol 8: 803-814, 2006.

22. Ravid D, Chuderland D, Landsman L, Lavie Y, Reich R and Liscovitch M: Filamin A is a novel caveolin-1-dependent target in IGF-I-stimulated cancer cell migration. Exp Cell Res 314: 2762-2773, 2008

23. Gawecka JE, Griffiths GS, Ek-Rylander B, Ramos JW and Matter ML: R-Ras regulates migration through an interaction with filamin A in melanoma cells. PLoS One 5: e11269, 2010.

24. Zhu TN, He HJ, Kole S, et al: Filamin A-mediated down-regulation of the exchange factor Ras-GRF1 correlates with decreased matrix metalloproteinase-9 expression in human melanoma cells. J Biol Chem 282: 14816-14826, 2007. 